\title{
TEM/STM system for study of nanowires and nanoparticles: How is it important to obtain 4D information of nanomaterials
}

Kunio Takayanagi

Tokyo Institute of Technology, Physics Department, 2-12-1-H-51 Oh-okayama, Meguro-ku, Tokyo, 152-8551 Japan

In recent years nano-materials-phases attract much interest in advancing nano-scale science and technology [1]. New materials-phases such as carbon nanotubes has already been applied for usage in nano-technology, and search for new phases is progressed in wave. Electron microscope community has developed aberration corrector systems not only for SEM but also TEM/STEM, which enable us to observe at high-resolution amplitude and phase of the scattered/diffracted electron waves by the specimen.

To study function of nano-materials-phases, we need specimen- responses by photons, ions, and electrons changing by time to time. Time-dependent electron microscopy of the states (structural-state, energy-state) of nano-materials-phases becomes much more important in the present-stage of electron microscopy. Particularly, time-dependent electron microscopy of light elements has strong demands from fundamental science and from industrial interests (fuel cell technology, organic EL, nano-device technology).

To answer for the present-stage demands, an aberration corrected high-resolution electron microscope for time-dependent study on 3D-structural and energy states of light elements is devised. Since the development has just started, we only show our grand design of the system that is based on our TEM-SPM experiments of the present-stage. In our TEM-STM system, we use a miniaturized STM that allow us to simultaneous observation of the structure and electronic conductance of nanowires. In TEM-AFM system, forces on nanowires induced by elastic and plastic deformations are clarified. A new system, SPM combined aberration corrected TEM/STEM, give us rich information on time, space, and energy. 
References:

[1]. K. Takayanagi et al: J.Ele.Micr. Phys. 52(2003) 49. 